\title{
Respuestas agudas al entrenamiento de fuerza con cargas pesadas y al entrenamiento mediante estiramiento sobre el rendimiento en squat jump y countermovement jump.
}

\section{Acute affects of strenght training from heavy loads and static stretching training on squat jump and countermovement jump.}

\author{
González Rave, J ose María \\ Laboratorio de entrenamiento deportivo. Universidad de Castilla la Mancha \\ Machado, Leandro \\ Laboratorio de biomecánica. Universidade do Porto \\ Navarro Valdivielso, Fernando J osé \\ Laboratorio de entrenamiento deportivo. Universidad de Castilla la Mancha \\ Vilas-Boas, J oao Paulo \\ Laboratorio de biomecánica. Universidade do Porto
}

\section{R e su m e n}

El objetivo de este estudio es examinar los efectos agudos del entrenamiento con cargas pesadas, y del estiramiento estático sobre sujetos no entrenados y determinar si tiene un efecto positivo sobre el rendimiento en SJ y CMJ. 20 sujetos divididos en dos grupos (grupo de fuerza n: 10 y grupo de estiramientos $\mathrm{n}: 10)$. El grupo de fuerza realizó 3 series de 3 repeticiones con la máxima carga que puede soportar en 3RM y 3 min descanso entre serie. En el grupo de estiramientos los sujetos realizaban 3 series de 3 ejercicios de estiramientos correspondientes a flexores de rodilla, extensores de cadera y gemelos manteniendo el estiramiento pasivo durante 15 segundos en cada ejercicio. SJ y CMJ fueron medidos antes y después del ejercicio. La altura de salto se incrementó significativamente en el SJ después de los ejercicios de estiramiento $(p<0.05)$. No hubo diferencias significativas en el resto de variables.

\begin{abstract}
The purpose of this study were to examine the acute effects of strength training using heavy loads and static stretching for 15 seconds on SJ and CMJ. A population sample of 20 subjects was studied which was divided into two groups (strength training (ST) $n=10$ - and static stretching training (SS) $n=10-$ ) ST group performed 3 sets of 3RM load with 3 min rest between all sets. SS group performed 3 stretching exercises, with each exercise held for 15 second repetitions. The stretches included a seated bilateral hamstring stretch; Standing unilateral quadriceps stretch and standing unilateral calf stretch. Vertical jump performance was measured before and after exercise. The height jump in SJ increased significantly after static stretching training $(p<.05)$. There was no significant difference between the rest of variables.
\end{abstract}

Palabras clave: efectos agudos, entrenamiento, fuerza, estiramiento, SJ, CMJ.

Key Words: acute, training, strength, stretching, SJ, CMJ.

Correspondencia/correspondence: Dr. José María González Ravé.

Laboratorio de entrenamiento deportivo. Universidad de Castilla la Macha. Campus "Antigua Fábrica de Armas, Avda Carlos III s/n - 45071 Toledo, Spain

E-mail: JoseMaria.Gonzalez@uclm.es 


\section{INTRODUCCION}

En poco tiempo han surgido numerosos estudios que muestran los efectos positivos de las respuestas agudas al entrenamiento de fuerza sobre el rendimiento en salto vertical (Baker, 2003; Young, Jenner y Griffiths,1998; Baker y Newton, 2005; Duthie, Young and Aitken,2002), este tipo de efecto recibe el nombre de potenciación postactiva y es definida como el incremento en la capacidad de un músculo después de haber realizado contracciones previas, así la potenciación postactiva se ha convertido en un método eficaz para el incremento de la fuerza explosiva (McBride, Nimphius y ericsson, 2005; Gullich y Schmidtbleicher, 1996; Scott y Docherty, 2001). Los principios de potenciación postactiva han sido aplicados a atletas para la mejora de su rendimiento tanto en entrenamiento como en competición (Scott y Docherty, 2001). Un método habitual de evaluación de la eficacia de la potenciación postactiva es el salto vertical (McBride, Nimphius y ericsson, 2005).

Así, hay estudios que muestran que la ejecución de contracciones isométricas de los extensores de rodilla y cadera se correlaciona positivamente con el incremento en la potencia explosiva de piernas incrementando así el salto vertical. (Burkett, Philips y Ziuraitis, 2005; Gullich y Schmidtbleicher, 1996, Moir et al, 2004). Históricamente, el test de salto vertical ha sido usado para evaluar las cualidades isoinerciales en la musculatura del miembro inferior (Logan et al, 2000). Varios métodos de entrenamiento de fuerza han sido desarrollados para la mejora de la fuerza explosiva, usando cargas pesadas o ligeras, pliometría o entrenamiento "balístico" (Smilios et al.,2005). El entrenamiento para la mejora de la potencia es comúnmente usado con cargas ligeras (40-60\% de $1 \mathrm{RM}$ ) que son realizadas a gran velocidad (Baker, 2001). El entrenamiento con cargas pesadas (cercanas a $1 \mathrm{RM}$ o $1 \mathrm{RM}$, entre el $80-100 \% 1 \mathrm{RM}$ ) y realizadas con pocas (1 a 3 ) repeticiones se acompaña de largos periodos de descanso e induce a incrementos en la potencia muscular. Los resultados del estudio de Young, Jenner y Griffiths (1998) sugieren que la inclusión de una serie de 5 RM en un calentamiento (4 minutos antes de un Countermovement Jump [CMJ]) podía mejorar el rendimiento en actividades cuyo factor de rendimiento principal sea la potencia de los extensores de piernas.

González-Ravé y Garcia-Coll (2006) mostraron resultados similares en un entrenamiento a corto plazo usando cargas concentradas (85-90\% 1RM) 2 días en semana. Duthie, Young and Aitken, (2002) examinó el rendimiento en SJ y concluye que el entrenamiento de contraste es muy ventajoso para la mejora de la potencia pero solo cuando los deportistas poseen altos niveles de fuerza. El estudio de Smilios et al (2005) sobre efectos a corto plazo del entrenamiento mostraba como el rendimiento en el CMJ se mejora cuando se alternan en el entrenamiento métodos balísticos con cargas moderadas (30-60\% de $1 \mathrm{RM})$.

Los efectos agudos sobre la mejora de la potencia han sido estudiados usando ejercicios de estiramientos. Sin embargo, tanto entrenadores como deportistas debe ser cuidadosos con el efecto adverso que realizar estiramientos tiene sobre el rendimiento en saltos 
González, J.M., Machado, L., Navarro, F.J., Vilas-Boas, J.P. (2006). Respuestas agudas al entrenamiento de fuerza con cargas pesadas y al entrenamiento mediante estiramiento sobre el rendimiento en squat jump y countermovement jump. Revista Internacional de Ciencias del Deporte. 4(2), 47-56.

http://www.cafyd.com/REVISTA/art4n4a06.pdf

verticales (Young y Elliot, 2001; Knudson et al, 2001; Wallmann, Mercer y McWhorter, 2005; Nelson, Cornwell y Heise, 1996). Los resultados de Young y Elliot 2001) acerca de los efectos agudos del estiramiento estático sobre la producción de fuerza explosiva muestran una disminución en el rendimiento de actividades en las que interviene el ciclo estiramiento acortamiento. Resultados coincidentes con los de Knudson et al (2001), que afirman como el estiramiento durante un calentamiento puede ser contraproducente en el rendimiento de un CMJ en sujetos físicamente activos. Similares resultados mostraban Nelson, Cornwell y Neise (1996) en el que tras un trabajo de estiramiento en extensores de cadera y flexores de rodilla disminuyó el rendimiento en SJ y CMJ.

Wallmann Mercer y McWhorter (2005) investigaron los efectos del estiramiento sobre el músculo gastrocnemio usando electromiografia y un test de salto vertical, concluyendo que el estiramiento tenía un efecto negativo sobre el salto vertical (5,6\%), aunque se incrementaba la actividad electromiografica (17,9\%).

Yamaguchi and Ishii (2005) demostraron que el estiramiento estático de 100 segundos a 30 minutos reduce el rendimiento muscular, si como ya hemos apuntado durante la introducción el estiramiento produce efectos negativos sobre la producción de fuerza Yamaguchi and Ishii (2005) sugieren que la potencia extensora de piernas después del estiramiento estático durante 30 segundos en el miembro inferior no difiere de aquellos sujetos que no hacen estiramientos, utilizando como muestra hombres físicamente activos y no atletas de competición.

El objetivo de este estudio es examinar los efectos agudos del entrenamiento con cargas pesadas, y del estiramiento estático sobre sujetos no entrenados y determinar si tiene un efecto positivo sobre el rendimiento en SJ y CMJ, comprobando los efectos resultantes de cada una de las tareas inmediatamente después del ejercicio, utilizando medidas pre y postest que evaluarán la efectividad de cada programa de entrenamiento, identificando quizás que programa es el más adecuado para mejorar la fuerza de estos sujetos en relación al salto vertical.

\section{METODO}

\section{Participantes}

20 hombres y mujeres voluntarios participaron en este estudio, divididos en 2 grupos:

- Grupo1: entrenamiento con cargas pesadas ( $\mathrm{n}=10$; edad $21.6 \pm 1.1$ años; altura $169.8 \pm 6.8 \mathrm{~cm}$; peso corporal $65.5 \pm 14.4 \mathrm{~kg})$.

- Grupo 2: estiramiento ( $\mathrm{n}=10$; edad $21.8 \pm 1.4$ años; altura $172.4 \pm 5.7 \mathrm{~cm}$; peso corporal $67.3 \pm 8.9 \mathrm{~kg})$.

Los participantes eran asignados al azar a uno de los grupos, dando previamente consentimiento informado a participar en el estudio. Todos los sujetos eran estudiantes de ciencias de la actividad física y el deporte de la Universidad de Porto (Portugal), y no 
González, J.M., Machado, L., Navarro, F.J., Vilas-Boas, J.P. (2006). Respuestas agudas al entrenamiento de fuerza con cargas pesadas y al entrenamiento mediante estiramiento sobre el rendimiento en squat jump y countermovement jump. Revista Internacional de Ciencias del Deporte. 4(2), 47-56.

http://www.cafyd.com/REVISTA/art4n4a06.pdf

participaban en ningún tipo de entrenamiento físico sistematizado. Ninguno de los sujetos había realizado sistemáticamente entrenamiento de fuerza o de flexibilidad cuando el estudio comenzó.

Todos los sujetos fueron advertidos acerca de que no modificaran sus hábitos de ejercicio físico, se les aconsejó que continuaran con su rutina normal. Ninguno de ellos tenía ninguna enfermedad de tipo

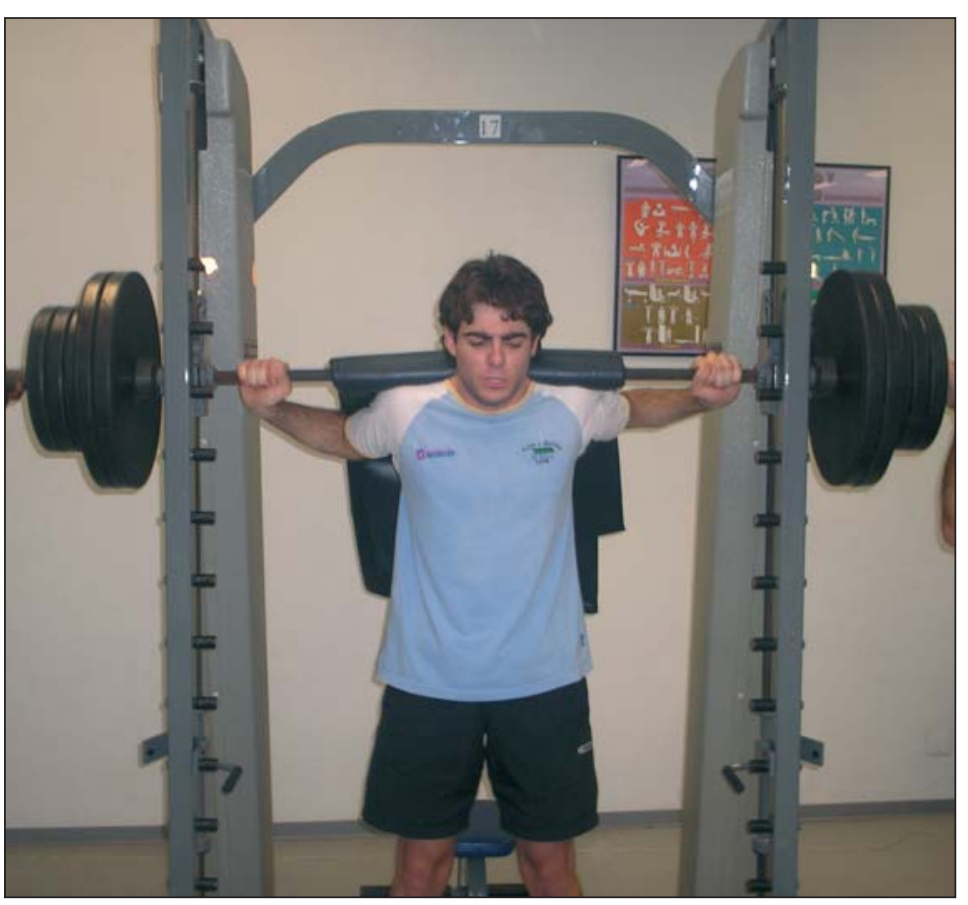

Figura 1.Realización de 3 RM con la máxima carga que puede soportar. el sujeto. músculo-esquelética.

Sesión de familiarización y test

\section{Sesión de familiarización}

Previo a las sesión de toma de datos, todos los participantes realizaron una sesión de familiarización con sus tareas, así el grupo de fuerza realizó un test para determinar con cuanta resistencia debían realizar 3RM en un semi-squat en una máquina "smith", en cada repetición, el sujeto debía alcanzar un ángulo en la rodilla de $90^{\circ}$. Se estableció un calentamiento estándar previo con 10 repeticiones con una carga estimada a este tipo de repeticiones. Para establecer la carga de 3 RM, los sujetos realizaron 3 repeticiones con una carga y si lo podían ejecutar correctamente se incrementaba el peso en 5-6 kg más dependiendo de las sensaciones del sujeto. En el grupo 1 realizaron 3 series de 3 repeticiones con una intensidad que correspondía a la carga de 3RM. En la figura $n^{\circ} 1$ mostramos a uno de los participantes del estudio con la carga correspondiente a su 3RM realizando el ejercicio con ayuda de 2 sujetos que controlan la carga. 
González, J.M., Machado, L., Navarro, F.J., Vilas-Boas, J.P. (2006). Respuestas agudas al entrenamiento de fuerza con cargas pesadas y al entrenamiento mediante estiramiento sobre el rendimiento en squat jump y countermovement jump. Revista Internacional de Ciencias del Deporte. 4(2), 47-56.

http://www.cafyd.com/REVISTA/art4n4a06.pdf

Todo el material de recogida de datos fue aportado por el laboratorio de biomecánica de la Universidad de Oporto, y la sala de acondicionamiento fue cedida por la Facultad de Ciencias del Deporte y la Educación Física de la citada Universidad.

\section{$\underline{\text { Sesión de test }}$}

Al realizar la sesión de recogida de datos del test, los sujetos realizaron un calentamiento estandarizado de 5 minutos en cicloergómetro antes de la recogida de datos. El rendimiento en SJ y CMJ fue evaluado usando una plataforma de fuerza (ByopacSystems- CA,USA) que registraba el tiempo de vuelo (seg.), y calculaba

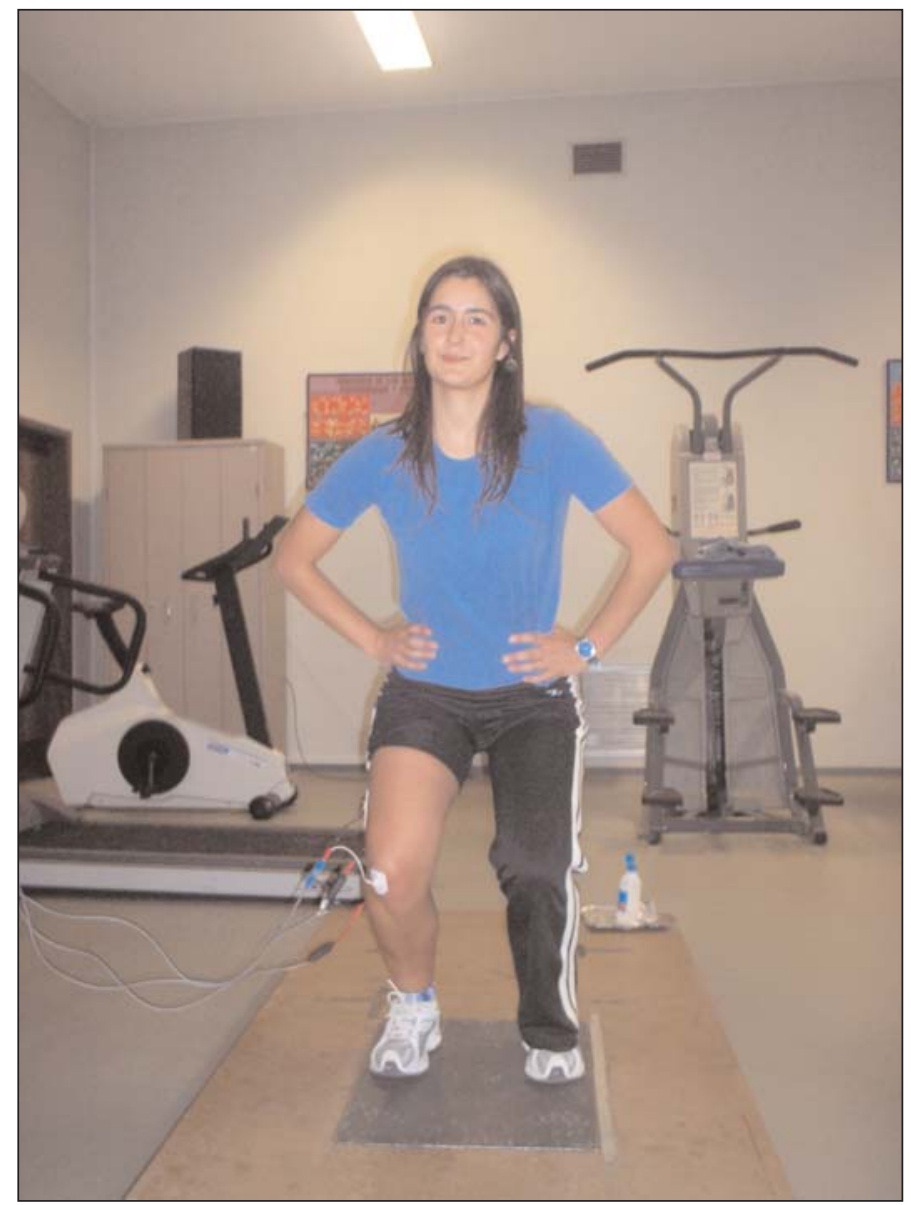

Figura 2. Realización del SJ en plataforma de fuerza. la altura de salto (cm.), tiempo de producción de fuerza (seg.) y producción de ésta (N.). Se realizaron los dos tipos de salto antes mencionados: Squat Jump (SJ) y Countermovement Jump (CMJ). En la figura numero 2 mostramos a uno de los participantes el estudio en la posición previa a la realización de un SJ.

Los participantes en la sesión de familiarización y de entrenamiento siempre estaban bajo la supervisión de dos especialistas en entrenamiento de fuerza que aseguraban la ejecución técnica correcta minimizando el riesgo de lesión. 
González, J.M., Machado, L., Navarro, F.J., Vilas-Boas, J.P. (2006). Respuestas agudas al entrenamiento de fuerza con cargas pesadas y al entrenamiento mediante estiramiento sobre el rendimiento en squat jump y countermovement jump. Revista Internacional de Ciencias del Deporte. 4(2), 47-56.

http://www.cafyd.com/REVISTA/art4n4a06.pdf

Para el análisis estadístico se utilizó el software SPSS 13.0 para Windows, y las siguientes técnicas de análisis: estadística descriptiva (tamaño de muestra, media, y desviación típica); prueba de Kolmogorov- Smirnov para comprobar la normalidad de la muestra y estadística inferencial mediante la prueba $\mathrm{T}$ de student para muestras relacionadas para la comparación pre-post y la prueba $\mathrm{T}$ de student para muestras independientes para la comparación entre Grupo 1 y Grupo 2 en las valoraciones pretest.

\section{RESULTADOS}

Antes de iniciar el entrenamiento comprobamos a través de la comparación usando la prueba $\mathrm{T}$ para muestras independientes como no existen diferencias significativas de entre ninguno de los dos grupos, es decir que los dos grupos inicialmente son iguales en cuanto a rendimiento en SJ y CMJ. Tras el tratamiento comprobamos como en SJ el grupo que utilizaba el entrenamiento de fuerza con cargas pesadas mejora $1 \mathrm{~cm}$ mientras que el grupo de flexibilidad mejora $2 \mathrm{cms}$. Sin embargo los resultados en CMJ indican como las mejoras son de $1 \mathrm{~cm}$ en ambos tratamientos. En la tabla $\mathrm{n}^{\circ} 1$ y 2 se presentan los resultados de altura en SJ y CMJ en ambos grupos, mostrándose un incremento significativo $(\mathrm{p}<0,05)$ en el SJ con el entrenamiento de flexibilidad mientras que en el resto de resultados no se observan variaciones estadísticamente significativas. Los resultados en cuanto a producción de fuerza en SJ muestran que el entrenamiento de flexibilidad aumenta la producción de fuerza en SJ, mientras que en el resto de valores la producción de fuerza disminuye. En la tabla $n^{\circ} 3$ y 4 se presentan los resultados obtenidos en producción de fuerza en SJ y CMJ en ambos grupos, no apreciándose variaciones estadísticamente significativas en ninguno de los dos grupos.

Tabla 1. Resultados del Countermovement Jump (media $\pm D T)$

\begin{tabular}{|c|c|c|c|}
\hline Entrenamiento & CMJ pretest & CMJ postest & Signif. $(*)$ \\
\hline Fuerza & $0.32 \pm 0.07 \mathrm{~m}$. & $0.33 \pm 0.06 \mathrm{~m}$. & NS \\
\hline Flexibilidad & $0.32 \pm 0.05 \mathrm{~m}$. & $0.33 \pm 0.06 \mathrm{~m}$. & NS \\
\hline
\end{tabular}

Tabla 2. Resultados del Squat Jump (media $\pm D T)$

\begin{tabular}{|c|c|c|c|}
\hline Entrenamiento & SJ pretest & SJ postest & Signif. $\left(^{*}\right)$ \\
\hline Fuerza & $0.28 \pm 0.06 \mathrm{~m}$. & $0.29 \pm 0.06 \mathrm{~m}$. & NS \\
\hline Flexibilidad & $0.28 \pm 0.04 \mathrm{~m}$. & $0.30 \pm 0.05 \mathrm{~m}$. & 0.05 \\
\hline
\end{tabular}

Tabla 3. Producción de fuerza (N) en Countermovement Jump (media $\pm D T)$

\begin{tabular}{|c|c|c|c|}
\hline Entrenamiento & CMJ pretest & CMJ postest & Signif. $\left(^{*}\right)$ \\
\hline Fuerza & $1659.65 \pm \mathrm{N}$. & $1621.15 \pm \mathrm{N}$. & NS \\
\hline Flexibilidad & $1543.33 \pm \mathrm{N}$. & $1511.67 \pm \mathrm{N}$. & NS \\
\hline
\end{tabular}

Tabla 4. Producción de fuerza ( $N$ ) en Squat Jump (media $\pm D T$ )

\begin{tabular}{|c|c|c|c|}
\hline Entrenamiento & SJ pretest & SJ set 3 & \multicolumn{2}{|c|}{ Signif. $\left(^{*}\right)$} \\
\hline Fuerza & $1661.38 \pm N$. & $1628.25 \pm N$. & NS \\
\hline Flexibilidad & $1530.8 \pm N$. & $1561.14 \pm N$. & NS \\
\hline
\end{tabular}




\section{DISCUSIÓN}

En este artículo pretendíamos comprobar los efectos agudos del entrenamiento con cargas pesadas, y del estiramiento estático sobre sujetos no entrenados y determinar si tiene un efecto positivo sobre el rendimiento en SJ y CMJ. Los resultados muestran como una tarea previa de entrenamiento previa utilizando el entrenamiento de fuerza de cargas pesadas con la máxima carga con la que puede realizar tres repeticiones no mejora de forma significativa el rendimiento en salto, ni la producción de fuerza tanto en un test de fuerza explosiva (SJ) como de fuerza elástico-explosiva (CMJ). Estos resultados no parecen coincidir con los expuestos por Young, Jenner y Griffiths (1998) que sugieren que la inclusión de una serie de 5 RM en un calentamiento (4 minutos antes de un Countermovement Jump [CMJ]) podía mejorar el rendimiento, ni con los estudios realizados con mujeres para la mejora de la fuerza máxima usando 2 sesiones de entrenamiento con cargas pesadas de González-Ravé y Garcia-Coll (2006) obteniendo mejoras en la fuerza dinámica máxima. Otros estudios en relación a la contracción isométrica de los grupos musculares pertenecientes a los extensores de rodilla y cadera correlacionan positivamente con el incremento en la potencia explosiva (Burkett, Philips y Ziuraitis, 2005; Gullich y Schmidtbleicher, 1996; Moir et al., 2004). En nuestro estudio hemos trabajado con contracciones anisométricas concéntricas y excéntricas, puediendo ser una causa concluyente por la que no se haya producido la mejora. Por otro lado, otra causa por la que no se haya producido la mejora puede deberse al tipo de población utilizada, ya que en la mayoría de los casos la muestra en otros estudios se corresponde con deportistas universitarios o de alta competición (Gullich y Schmidtbleicher, 1996; Duthie, Young and Aitken,2002; Young, Jenner y Griffiths, 1998) mientras que en nuestro estudio los participantes se corresponden con sujetos físicamente activos, lo que puede haber generado esa disminución en la producción de fuerza tanto en SJ como en CMJ, aunque ésta no haya sido significativa. Podemos afirmar, coincidiendo con Scott y Docherty, (2001) como los principios de potenciación postactiva solo pueden ser aplicados a atletas para la mejora de su rendimiento tanto en entrenamiento como en competición, y no a otro tipo de poblaciones como sujetos físicamente activos.

En este sentido, la utilización de cargas moderadas en el entrenamiento de fuerza quizás habría dado como resultado la mejora en el rendimiento del salto, tal y como afirman Smilios et al (2005), y sobre todo tratándose de sujetos no adaptados al entrenamiento de fuerza.

En relación al entrenamiento mediante el uso de estiramientos de 3 grupos musculares implicados en el salto durante 15 segundos cada uno comprobamos como se ha producido un incremento significativo $(p<0,05)$ en el rendimiento del SJ con el entrenamiento de flexibilidad, aunque no en el CMJ. Estos resultados en CMJ pueden deberse a una reducción del nivel de actividad neuromuscular y los cambios mecánicos asociados a las propiedades viscoelásticas del tendón son los causantes de la disminución del rendimiento en los ejercicios de stretching como afirman Yamaguchi e Ishii (1998) y Kokkonen et al, (2005). Por otra parte, Unick et al (2005) presenta como los efectos agudos del estiramiento estático y balístico pueden no afectar de forma 
adversa el rendimiento en mujeres entrenadas (jugadoras de baloncesto) por lo que se podría explicar la mejora en el SJ.

Sin embargo, tanto entrenadores como deportistas debe ser cuidadosos con el efecto adverso que realizar estiramientos tiene sobre el rendimiento en saltos verticales (Young y Elliot, 2001; Knudson et al, 2001; Wallmann, Mercer y McWhorter, 2005; Nelson, Cornwell y Heise, 1996), aunque como afirman Yamaguchi e Ishii (1998), quizás sea una cuestión del tiempo dedicado al ejercicio en cuestión, ya que si es de 100 segundos a 30 minutos reduce el rendimiento muscular. En el caso específico de nuestro estudio solo ha sido de 15 segundos en cada ejercicio en cada uno de los 3 ejercicios de estiramientos correspondientes a flexores de rodilla, extensores de cadera y gemelos por lo que no afectaría de forma adversa el rendimiento.

En relación a la producción de fuerza no hay resultados concluyentes. En este estudio ha aumentado la producción de fuerza donde el rendimiento en altura en SJ y CMJ no ha sido estadísticamente significativo, y hadisminuido la producción de fuerza incrementándose el rendimiento en SJ y CMJ. Este comportamiento en relación a la producción de fuerza- altura de salto no podemos explicarlo por si solo con los datos que tenemos, deberíamos haber tenido información adicional a través del análisis de la actividad electromiografica como en el estudio de Wallmann Mercer y McWhorter (2005) comprobando la influencia de la actividad neuromusucular de la musculatura que interviene en el salto y a que es debida ésta.

En conclusión, podemos afirmar que las respuestas agudas al entrenamiento de fuerza con cargas pesadas no mejora la producción de fuerza ni el rendimiento en salto vertical medido en SJ y en CMJ, mientras que las respuestas agudas al entrenamiento de flexibilidad estática durante 15 segundos mejora significativamente $(\mathrm{p}<.05)$ el rendimiento en salto del SJ y la producción de fuerza aunque no de forma significativa.

\section{AGRADECIMIENTOS}

Quisiéramos agradecer al Dr. Filipe Almeida Viana da Conceicao, profesor de atletismo en la FCDEF de la Universidade do Porto su ayuda y disponibilidad para que todo saliera bien en la recogida de datos. Muito Obrigado. 


\section{REFERENCI AS BI BLI OGRÁFI CAS}

Baker, D. (2003) Acute effect of alternating heavy and light resistances on power output during upper-body complex training. J. Strength Cond. Res. 17: 493497.

Baker, D. and R.U. Newton.(2005) Acute effect on power output of alternating an agonist and antagonist muscle exercise during complex training. J. Strength Cond. Res. 19: 202-205.

Duthie, G.M., W.B. Young, and D.A. Aitken. (2002) The acute affects of heavy loads on jump squat performance: an evaluation of the complex and Contrast methods of power development. J. Strength Cond. Res. 16: 530-538.

Logan, P., Formasiero, D., Abernethy, P., Lynch, K. (2000) Protocols for the assessment of isoinertial strength. In: Physiological tests for elite athletes. CJ Gore and Australian Institute of Sport. Eds. Champaign, IL: Human Kinetics. pp 200-222.

Baker, D. (2001) Acute and long term power responses to power training: observations on the training of an elite power athlete. Strength Cond. J. 23:4756.

McBride, J.M., S. Nimphius, and T.M. Erickson. (2005)The acute effects of heavyload squats and loaded countermovement jumps on sprint performance. J. Strength Cond. Res. 19(4): 893-897.

Burkett, L.N., W.T. Philips and J. Ziuraitis. (2005)The best Warm-up for the vertical jump in college-age athletic men. J. Strength Cond. Res. 19:673-676.

Gullich, A. and D. Schmidtbleicher. (1996) MVC-induced short term potentiation of explosive force. New Stud. Athletics. 11: 67-81.

Moir, G., C. Button, M. Glaister, and M.H. Stone, (2004) Influence of familiarization of the reliability of vertical jump and acceleration sprinting performance in physically active men. J Strength Cond Res 18: 276-280.

González-Ravé, J.M. and V. Garcia-Coll. (2006) Respuestas agudas al entrenamiento de fuerza máxima en deportistas femeninas. Arch. Med. Dep. (EN PRENSA).

Knudson, D., K. Bennett, R. Corn, D. Leick and C. Smith. (2001) Acute effects of stretching are not evident in the kinematics of the vertical jump. J. Strength Cond. Res. 15(1): 98-101.

Nelson, A.G., A. Cornwell and G. Heise. (1996) Acute stretching exercises and vertical jump stored elastic energy. Med. Sci. Sports Exerc. 28(suppl.) : 101.

Kokkonen, J., A.G. Nelson, and A. Cornwell. (1998) Acute muscle stretching inhibits maximal strength performance. Res Q. Exerc. Sport. 69:411-415.

Moir, G., C. Button, M. Glaister, and M.H. Stone. (2004) Influence of familiarization of the reliability of vertical jump and acceleration sprinting performance in physically active men. J. Strength Cond. Res. 18(2): 276-80.

Scott, S.L. and D. Docherty. (2004) Acute effects of heavy preloading on vertical and horizontal jump performance. J. Strength Cond. Res. 18(2):201-205. 
Smilios, I., Pilianidis, T., Sotiropoulos, K., Antonakis, M., Tokmakidis, S. (2005) Short term effects of selected exercise and load in Unick, J., H.S. Kieffer, W. Cheesman, and A. Feeney. (2005) The acute effects of static and ballistic stretching on vertical jump performance in trained women. J. Strength Cond. Res. 19(1):206-212. ontrast training on vertical jump performance. J. Strength Cond. Res. 19: 135-139.

Wallmann, H.W., J.A. Mercer, and J.W. McWhorter. (2005) Surface electromyographic assessment of the effect of static stretching of the gastrocnemius on vertical jump performance. J. Strength Cond. Res. 19(3): 684-688.

Yamaguchi, T., and K. Ishii. (2005)Effects of static stretching for 30 seconds and dynamic stretching on leg extension power. J. Strength Cond. Res. 19(3):677683.

Young, W. and S. Elliot. (2001) Acute effects of static stretching, propioceptive neuromuscular facilitation stretching, and maximum voluntary contractions on explosive force production and jumping performance. Res. Q. Exerc. Sport 72: 273-279.

Young, W.B., A. Jenner, and K. Griffiths. (1998) Acute enhancement of power performance from heavy load squats. J. Strength Cond. Res. 12: 82-84. 